\title{
TEORIA EGO-ECOLÓGICA E O ESTUDO DA IDENTIDADE SOCIAL - APLICABILIDADE EM PESQUISAS DE ENFERMAGEM
}

\author{
The ego-ecological theory and the study of the social identity applied to nursing \\ research \\ Teoría egoecológica y el estudio de la identidad social - aplicabilidad en la investigación \\ en enfermería
}

Antônia Regina Ferreira Furegato ${ }^{2}$

\begin{abstract}
RESUMO
A enfermagem vem trilhando caminhos teórico-metodológicos mais sensíveis para desenvolver um conhecimento que discuta, analise e fundamente sua prática cotidiana. 0 objetivo deste texto foi refletir sobre as bases metodológicas e a aplicação da Teoria Ego-ecológica nas pesquisas em enfermagem. Por este caminho, é possível conhecer a identidade do indivíduo, suas peculiaridades e sua realidade, através das representações que ele possui acerca de si mesmo, de seus grupos de pertencimento e da sociedade. A Teoria Ego-ecológica entende a identidade como um modo de construção da realidade, a partir do qual o mundo exterior fundese ao mundo interior a partir da história de vida do indivíduo. A análise Ego-Ecológica permite compreender o cliente e suas complexidades e paradoxos e as relações que estabelece no contexto da família, do trabalho, do lazer e nas situações sociais no espaço micro ou macrossocial.
\end{abstract}

Palavras-chave: Pesquisa em enfermagem. Pesquisa qualitativa. Relações interpessoais. Identificação social.

\begin{abstract}
Nursing has been through theoretical and methodological paths more sensible to the development of a kind of knowledge that is able to discuss, to analyze and provide a basis to its daily practice. This text aims to discuss the methodological basis and the application of the ego-ecological theory in nursing research. According to this theory it is possible to know the individual's identity, its peculiarities and its reality, by means of his/her selfrepresentations and the representations (s)he has about his/her social groups. The ego-ecologic theory sees identity as a reality construct in which the outer world and the inner world merge in face of the individual history. The ego-ecological analysis allows one to understand the client and his/her complexities and paradoxes and the relations established on family, work, leisure and micro-social and macro-social levels.
\end{abstract}

Keywords: Nursing research. Qualitative research. Interpersonal relations. Social identitification.

\section{Resumen}

La enfermería pasa por caminos teóricos y metodológicos más sensibles para desarrollar los conocimientos para discutir, analizar y fundamentar su práctica cotidiana. El objetivo de este texto es reflexionar sobre las bases metodológicas y la aplicación de la Teoría Egoecológica de investigación en enfermería. A través de esta forma, es posible conocer la identidad del individuo, su propia cultura y realidad a través de las representaciones que tiene sobre símismo, su pertenencia a grupos y la sociedad. La teoría Ego ecológica comprende la identidad como una forma de construir la realidad, desde que el mundo exterior venga a unirse con el mundo interior de la historia de vida de la persona. El análisis Ego Ecológica permite comprender al cliente y sus complejidades y paradojos y las relaciones que se establecen dentro de la familia, del trabajo, del ocio y las situaciones sociales en el espacio micro o macro social.

Palabras clave: Investigación en enfermería. Investigación cualitativa. Relaciones interpersonales.Identificación social.

'Doutora em enfermagem psiquiátrica; Professora Adjunta do Departamento de Enfermagem Médico-Cirúrgica da Escola de Enfermagem Alfredo Pinto/ UNIRIO. Rio de Janeiro- RJ. Brasil. E-mail: rosane.dv@gmail.com ;'Doutora em enfermagem psiquiátrica; Professora Titular do Depto. de Enfermagem Psiquiátrica e Ciências Humanas da EERP/USP. Ribeirão Preto - SP. Brasil. E-mail: furegato@eerp.usp.br 


\section{INTRODUCÃO}

Ao longo do tempo, a enfermagem vem trilhando vários caminhos teórico-metodológicos e continua buscando métodos mais sensiveis para desenvolver um conhecimento que discuta, analise e fundamente a prática cotidiana da enfermagem. Neste sentido, acredita-se que a prática (ciência), e a arte (criatividade) constituem a força da enfermagem à procura de novos paradigmas, novos rumos e de novas linguagens, inclusive na arte de pesquisar. ${ }^{1}$

Este estudo apresenta a proposta metodológica de Marisa Zavalloni², psicóloga social canadense. Trata-se do referencial teórico-metodológico "Teoria ego-ecológica", que é uma das vertentes da Teoria das Representações Sociais. Através desta metodologia, é possível conhecer a identidade do indivíduo, suas peculiaridades e sua realidade, por meio das representações que ele possui acerca de si mesmo, assim como do seu grupo de pertencimento. Vale destacar que a Teoria Ego-Ecológica possibilita a compreensão mais profunda não só do indivíduo que precisa de ajuda, mas também de todo 0 ambiente histórico e cultural envolvido no processo saúde/ doença, otimizando o cuidar em enfermagem.

0 objetivo, portanto, deste texto é apresentar uma reflexão sobre as bases metodológicas para a aplicação da Teoria Ego-ecológica nos estudos e pesquisas em enfermagem.

\section{A ENFERMAGEM E A TEORIA DAS REPRESENTAÇÕES SOCIAIS}

A investigação científica no Brasil iniciou-se com a criação da Escola de Enfermagem do Departamento Nacional de Saúde Pública (DNSP), em 1923. Concretizou-se em 1932 com a criação do primeiro veículo de divulgação de informações de enfermagem, os "Annaes de Enfermagem", que desde 1954 passou a se chamar Revista Brasileira de Enfermagem REBEn. ${ }^{3}$

A Associação Brasileira de Enfermagem (ABEn) teve importante papel, organizando, entre 1956 e 1958, o primeiro estudo sistematizado que recebeu o título de "Levantamento de Recursos e Necessidades em Enfermagem”. Esta investigação foi realizada com o apoio da Fundação Rockefeller, da Organização Mundial de Saúde (OMS), Ministério da Educação, Ministério da Saúde, Comissão Nacional de aperfeiçoamento de Pessoal de Nível Superior (CAPES) e Serviço Especial de Saúde Pública (SESP). ${ }^{4}$

Outros fatos importantes marcaram a história da pesquisa em enfermagem no Brasil, como a Reforma Universitária em 1968 que indicava e ratificava a importância da associação do ensino com a pesquisa, assim como a criação do primeiro curso de pós-graduação em nível de mestrado, na Escola de Enfermagem Anna Nery, em 1972. ${ }^{3}$

Com o apoio da Fundação de Apoio à Pesquisa de São Paulo (FAPESP) foi realizado um minucioso estudo sobre a criação e o desenvolvimento da pós-graduação no Brasil e seus reflexos na formação de pesquisadores para todo o território nacional e a criação e consolidação de Núcleos de pesquisa na enfermagem brasileira. ${ }^{5}$ Observa-se que a pós-graduação teve influência marcante na produção do conhecimento pelas enfermeiras.

Nas décadas de 1950 e 1960, as pesquisas de enfermagem no Brasil focalizavam o ensino e a profissão, as necessidades sentidas pelos pacientes, a avaliação da assistência de enfermagem, o estudo de métodos para melhorar a qualidade do cuidado e o rendimento das atividades. ${ }^{6}$ Até a década de 1980, o foco estava na metodologia de assistência em enfermagem, necessidades do paciente, ensino, avaliação da assistência, a profissão, a equipe de enfermagem, epidemiologia e biologia.

Levantamento realizado em periódicos de enfermagem entre os anos 1990 e 2000, na área de saúde da mulher, revelou tendência para os estudos baseados em técnicas qualitativas, voltadas para 0 aspecto assistencial. ${ }^{6} \mathrm{As}$ abordagens metodológicas mais comumente utilizadas por enfermeiros foram as Representações Sociais e a Fenomenologia. Neste sentido, faz-se importante destacar que a abordagem qualitativa possibilita maior aproximação entre 0 pesquisador e o pesquisado, facilitando uma relação dialógica entre os profissionais e seus clientes, ampliando o conhecimento dos aspectos culturais da população. ${ }^{6}$

0 enfermeiro lida diariamente com sentimentos, dores e angústias dos clientes e, muitas vezes, está inserido em um contexto de trabalho pobre de recursos que o auxiliem neste cuidado. A pesquisa é o instrumento indicado para observar de forma sistematizada a especificidade e a complexidade do ser humano, facilitando a prática do cuidado e possibilitando o desenvolvimento de concepções e ações mais adequadas às especificidades do trabalho do enfermeiro.

0 enfermeiro, ao entrar em contato com a subjetividade e a peculiaridade de sua clientela, tenta estabelecer uma relação terapêutica com o cliente, pois o espaço do cuidar é atravessado tanto pelo cotidiano do cliente quanto pelos seus sentimentos, saberes e hábitos de vida. ${ }^{7}$

A aproximação dos pesquisadores da enfermagem com as técnicas qualitativas e, consequentemente, das representações sociais tem coerência com uma prática matizada por tons humanísticos. Desta forma, o profissional pode perceber o cliente de forma integral, inserido no contexto social, cultural e político, o que possibilita a construção de um cuidado mais adequado às necessidades do cliente., ${ }^{6,7}$ Neste sentido, 0 enfermeiro precisa conhecer melhor os fenômenos históricosociais, de forma tal que articule o saber e o fazer, tendo como ponto central o cliente no seu contexto psicossocial.

0 cuidado em enfermagem não deve ser visto como algo apenas técnico, superficial, repetitivo e generalizável, mas como algo denso, complexo e principalmente singular. Os enfermeiros têm por base o que cada sujeito sente, pensa, 
vivencia, deseja e sofre de forma única, pois cada um vive a sua própria história, em seu próprio ambiente ecológico. ${ }^{8} 0$ ambiente ecológico inclui as relações sociais, culturais, políticas e a subjetividade que é peculiar a cada sujeito inserido em seus vários contextos.?

\section{A ABORDAGEM TEÓRICO-METODOLÓGICA EGO-ECOLÓGICA E O ESTUDO DA IDENTIDADE SOCIAL}

Marisa Zavalloni é estudiosa da área das representaç̃̃es sociais e professora da Universidade de Montreal, no Canadá. Suas publicações iniciaram-se na década de 1960, tendo inclusive produzido textos em conjunto com Moscovici, Doise e outros pesquisadores das representações sociais. Na década de 1980, publicou vários textos em um referencial teórico-metodológico que intitulou Teoria Egoecológica. Por meio deste referencial, a autora entende o indivíduo não como um receptor passivo, mas como um participante ativo de seu mundo. Apresenta o indivíduo inserido em um projeto matizado por lutas e por conflitos, onde ele participa da situação em questão, transformando e sendo transformado. ${ }^{9}$

Através da linguagem é possível alcançar maior compreensão do sujeito, pois pode ser usada como um instrumento de ação e de reprodução de uma determinada ordem social. 0 estudo da linguagem permite observar sistemas classificatórios que expressam sentimentos e pensamentos relativos à identidade do indivíduo e seus valores culturais. Vale destacar que através da linguagem o indivíduo expressa sua percepção acerca de sua identificação ou diferenciação de aspectos como etnia, gênero, classe, religião, entre outros. Cada grupo desenvolve um vocabulário para marcar sua identidade e se diferenciar dos outros e, desta forma, elaborar novos significados constantemente. Os significados atribuídos às palavras são utilizados para ampliar o vínculo da linguagem a uma experiência ou a um projeto específico. ${ }^{11}$

A interação de uma pessoa com seu meio social pode ser deduzida ou mensurada como produto de uma realidade exterior. Entretanto, o resultado final estaria incompleto, sendo então necessário considerar o interior do indivíduo, representado através das palavras e seus significados que tanto podem estar cristalizados ou em processo de transformação ou atualização. ${ }^{2}$

As representações não são somente ideias ou a tradução mental de uma realidade exterior, mas refletem o espaço imaginário e simbólico do indivíduo. As representações sociais são criações que expressam valores e concepções da pessoa sobre si mesma, sobre as pessoas com as quais convive e sobre a sociedade, sendo este o objeto de estudo da Teoria ego-ecológica. As representações, estando vinculadas às palavras e aos temas, possuem uma ressonância particular, captando aspectos relativos à identidade, às experiências, lembranças e imagens pessoais. ${ }^{2}$
A partir da Ego-ecologia busca-se entender a maneira como um ambiente exterior específico é constituído e como se organiza em um ambiente interior. Este método busca entender como se constroem as diferentes realidades e as regras que geram as motivações e ações da pessoa.?

A identidade surge como o lugar de encontro do individual e do social, do objetivo e do subjetivo. É um método indicado para estudar a mediação entre o meio exterior e o interior e a identidade é percebida como o produto da história pessoal e individual, que reflete as experiências passadas. Pela Teoria Ego-ecológica, a identidade é compreendida como um modo de construção da realidade, a partir do qual o mundo exterior é introjetado em função de um projeto e de uma história particular. A identidade é o produto da interação do sujeito com o mundo que, em contrapartida, também influencia o seu próximo. ${ }^{9}$

A Ego-ecologia compreende uma dinâmica que permite conhecer a construção e a reconstrução da identidade através de três dimensões interligadas, ou seja, as representações que um indivíduo possui acerca de si mesmo, dos outros (indivíduos ou grupos) e da sociedade.?

Para que se tenha uma representação mais abrangente do individuo é necessário que ele seja percebido como resultado de sua interação com os outros e com a sociedade. 0 senso que a pessoa elabora sobre 0 outro tem ligação direta com as relações imaginárias e reais que possui acerca de si mesma. ${ }^{10}$

A Ego-ecologia procura compreender as representações do sujeito a partir do ambiente interior, através da maneira como os conteúdos são organizados na memória, as lembranças pessoais, as imagens e as experiências. 0 ambiente interior reflete as situações concretas, do mesmo modo que as situações imaginárias refletem as relações com os outros, com a sociedade, sobre o desejo e a probidade. Esta análise permite conhecer o microcosmo do indivíduo e suas regras, através das representações que ele constrói sobre cada tema. ${ }^{2}$

A Ego-ecologia situa-se no pensamento consciente ou pré-consciente, quer dizer, nas representações e nas lembranças pessoais, imagens ou experiências que surgem da memória. Neste sentido, a memória seria o reservatório do pensamento consciente. É o que a autora chama de memória emocional do mundo. ${ }^{2}$

0 método de busca dessas memórias é ativo e, através das palavras e opiniões do individuo, identifica as representações acerca de si mesmo, das pessoas que o cercam e dos diversos grupos nos quais está inserido; permite conhecer atributos (positivos ou negativos) construídos sobre o tema pesquisado, bem como os graus de importância dentro do seu sistema de valores.?

\section{ANÁLISE EGO-ECOLÓGICA: O MÈTODO}

0 sistema de levantamento de dados desenvolve-se em três etapas, tendo como ponto de partida o entendimento de que toda pessoa está inserida em uma matriz social, fazendo parte, concomitantemente, de diferentes grupos sociais e culturais. Os grupos dos quais a pessoa faz parte constituem- 
se em categorias onde se inscreve a identidade social objetiva e o delineamento de perfil do sujeito. Estas categorias são obtidas através do grau de identificação ou dissociação do sujeito com os membros do grupo e/ou da sociedade na qual está inserido. ${ }^{2}$

A primeira etapa (subdividi-se em três fases) primeiramente se obtém de forma sistemática, através da complementação de frases, da representação que o sujeito faz de si mesmo e dos diferentes grupos aos quais pertence. Fazse necessário destacar que as frases devem ser completadas a partir de dois estímulos distintos ("Nós" e "Eles"), tal como nos exemplos abaixo:

- "Como enfermeiras, nós..."; "Como mulheres, nós..."

- "Como enfermeiras, elas..."; "Como mulheres, elas..."

Este duplo estímulo (nós e eles) tem por objetivo gerar certo afastamento do entrevistado do objeto de representação, além de facilitar o julgamento crítico de si mesmo e de seus pares.

0 material expresso através de frases ou de palavras compõe a Identidade Social Subjetiva, através da qual se observam aqueles aspectos dos membros dos grupos com os quais o sujeito se identifica ou se diferencia.

Após a complementação de frases, o entrevistado atrela os conteúdos citados à própria pessoa através da autoinclusão ou da autoexclusão. Tal classificação gerará uma identificação especifica com os aspectos sociais, culturais, históricos, psicossociais e/ou políticos do objeto de investigação. Neste momento, pergunta-se ao entrevistado se ele se identifica (autoinclusão) ou não (autoexclusão) com os conteúdos citados por ele.

No terceiro momento, retorna-se ao conteúdo produzido e solicita-se ao entrevistado que classifique as características em atitudes positivas ou negativas, para observar os campos simbólicos onde se depositam as realizações, as atitudes, os projetos e os desejos do indivíduo.

Através do levantamento dos temas ou das palavraschave são construídas as imagens do grupo e, ao mesmo tempo, é possível compreender a percepção que o indivíduo possui acerca de ser, ter e fazer.

A segunda etapa tem por objetivo elucidar e aprofundar o estudo acerca das representações sociais que estão sendo investigadas. De forma sistemática busca-se compreender a história do indivíduo, assim como sua relação com os atributos citados. Esta busca pode ser realizada através de perguntas, como por exemplo, o que vem à sua mente quando pensa acerca do conteúdo citado, ou ainda a descrição do grupo que mais se assemelha a ele e do grupo que mais se diferencia. Dos conteúdos obtidos na coleta destes dados, o pesquisador pode destacar:?

1) 0 que o sujeito atribui a si mesmo;

2)As referências implícitas sobre os grupos, sobre as pessoas ou sobre as imagens nas quais ele reflete sua própria identificação a respeito do objeto investigado;

3)A atribuição de valor (positivo ou negativo) a cada um dos significados no que concerne ao grupo e ao indivíduo.

Procura-se ordenar este material, tomando-se por base um sistema de identificação e de oposição binária considerandose três aspectos:?

- Eux Outro;

- Bom x Mau;

- Importante x Não importante.

A análise dos conteúdos expressos pelos sujeitos conduz a quatro possíveis categorizações da identidade do indivíduo:

I. Identificação positiva com o grupo => Valorização das qualidades intrínsecas e extrínsecas do objeto investigado;

II. Diferenciação positiva com o grupo => Valorização dos aspectos diferenciais;

III.Identificação negativa com o grupo => Desvalorização intrínseca e extrínseca ligada a privação ou vitimização;

IV.Diferenciação negativa com o grupo => Desvalorização dos aspectos intrínsecos que se apresentam como oposição, causando privação ou ameaça.

Esta análise contextualizada do ambiente histórico e social oferece indicadores do quanto a pessoa está impregnada pelo sistema de significação deste contexto. A partir daí, o pesquisador observa se há diferenciação positiva ou negativa com o objeto do estudo, possibilitando a construção de categorias que indicam uma aproximação ou afastamento entre a identidade do entrevistado e a identidade do grupo em que ele está inserido. Vale ressaltar que, ao compreender a percepção do indivíduo a respeito dos integrantes de um grupo, é possivel conhecer as características essenciais que o indivíduo manifesta a respeito de si mesmo (tanto aquelas com as quais se identifica como aquelas das quais se dissocia).

A terceira etapa é intitulada "Contextualização Representacional". São os procedimentos de retomada de cada palavra e de sua exploração junto com o próprio sujeito da pesquisa. $^{2}$

Nesta fase da análise, são percorridos os conteúdos coletivos (história do grupo, projetos e privações sociais) e os conteúdos individuais (biografia, projetos e estratégias de adaptação) sobre o tema focalizado.

São feitas perguntas sobre o significado do conteúdo para aquele grupo, justifica-se aquela associação ao grupo, situações particulares são lembradas, entre outros.

A análise do conteúdo obtido nesta etapa permite que aspectos da identidade sejam observados e analisados. Os seguintes critérios para construção de eixos de identidade são 
definidos: identificação positiva com o grupo; identificação negativa com o grupo; diferenciação positiva com o grupo; e diferenciação negativa com o grupo. Nesta fase, o pesquisador não realiza nenhum tipo de inferência, pois o próprio entrevistado já classificou suas respostas. Após a alocação dos atributos informados nos eixos, observa-se através da contagem simples o quantitativo de respostas em cada quadrante do eixo. 0 resultado permite estabelecer o grau de identificação dos indivíduos com seus pares, com os indivíduos de outros grupos com os quais convive e com a sociedade. ${ }^{9}$

\section{RESULTADOS}

A organização das respostas em eixos, baseia-se na valoração dos atributos feita pelos próprios entrevistados, no momento em que completaram as frases. Os termos egomórfico e alomórfico são utilizados como indicadores do grau de identificação do indivíduo com o atributo citado por ele. 2,9,10

0 termo "egomórfico" (características aplicadas a si mesmo) é utilizado para indicar que o entrevistado se identifica com o atributo que referiu para o sujeito ou situação em questão. Por sua vez, o termo "alomórfico" (características dissociadas de si mesmo) é aplicado quando o sujeito informa não se identificar e se dissocia das características destacadas por ele e que se relacionam com o sujeito ou situação referida. ${ }^{2}$

Vale a pena ratificar que além de informar se há identificação ou não com o atributo citado, o entrevistado também classifica suas respostas em positivo ou negativo. Foram desenvolvidos parâmetros e quadros que apontam alguns caminhos possíveis para o desenvolvimento da análise dos dados na Teoria Ego-Ecológica. ${ }^{8}$ Através da contagem dos atributos em cada eixo inicia-se a análise dos dados propriamente dita. 0 Quadro 1 foi construído como parâmetro de análise das respostas ${ }^{8: 110}$, intitulado pela autora como "Espaço Elementar de Identidade Social". Este quadro de análise diz respeito à maneira como o entrevistado compreende sua relação com o seu par ou com um determinado grupo, tendo o centro deste construto 0 objeto de representação:

Quadro 1. Espaço Elementar de Identidade de Social ${ }^{2}$

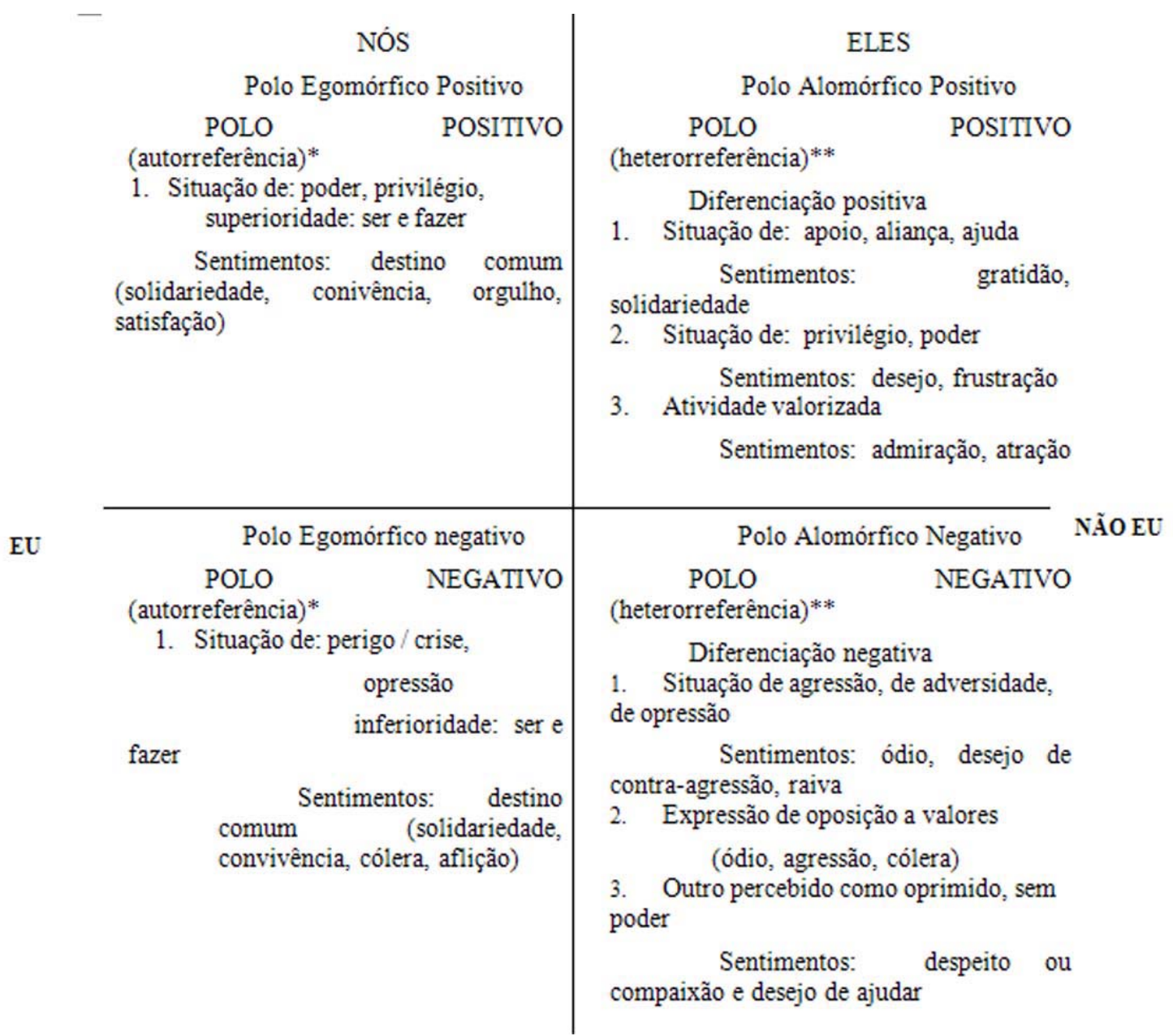


Há quatro maneiras de proceder à avaliação das respostas dos entrevistados, de acordo com a distribuição dos resultados obtidos nos eixos do Espaço Elementar de Identidade Social: ${ }^{2}$

A-Se a maioria das respostas ('Nós' e 'Eles') se encontra nos polos egomórficos, há uma importante identificação do sujeito com o grupo. A codificação do grupo evoca dimensões ou atitudes similares às do grupo. 0 sujeito tem uma tendência a ter uma visão positiva de si mesmo, do grupo e em relação ao objeto pesquisado;

B-Se há preponderância das respostas referentes a 'Nós' nos polos egomórficos, da mesma forma em que há preponderância das respostas referentes a 'Eles' nos polos alomórficos, isto indica a existência de subgrupos. 0 sujeito estabelece aproximação ou afastamento dos subgrupos, de acordo com sua identificação. Neste caso, 'Nós' indica as qualidades positivas e 'Eles' os defeitos, os traços rejeitados e os contravalores;

C-Se a maioria das respostas relativas a 'Nós' se encontra nos polos alomórficos e relativas a 'Eles' nos polos egomórficos, isto indica um código em que 'Eles'evoca aspectos ideais do grupo, enquanto 'Nós' evoca os limites e as falhas. Neste caso, as características positivas estão relacionadas a 'Eles' e as negativas, a 'Nós';

D-Se a maioria das respostas ('Nós' e 'Eles') se situa nos polos alomórficos, há indicação de distanciamento do sujeito entrevistado com o grupo em estudo. Neste caso, frequentemente 'Nós' e 'Eles' são codificados como subgrupos, diferentes do próprio sujeito. Tanto os aspectos positivos como os negativos podem afetar o sujeito, dependendo do significado atribuído à identidade do outro.

Observa-se que este método permite utilizar diferentes eixos de significação e análise, explicitando o valor de cada palavra trabalhada, procurando desdobrar cada eixo temático em torno das representações elaboradas. Esta característica permite que, todas as vezes que seja retomada a análise, ocorra a geração de novas dimensões passíveis de serem exploradas, quer seja através do aspecto grupal, individual, histórico, social, cultural ou político.

Através do estudo da identidade social, há a proposta de quatro dimensões psicológicas relevantes para a compreensão das representações sociais do sujeito em estudo: ${ }^{11}$

- As representações ligadas a um saber referente à identidade do indivíduo e do(s) grupo(s) em estudo;

- A articulação entre as representações do grupo e as do próprio individuo;

- Os limites do conceito de grupo

- A percepção do entrevistado em uma situação social complexa.

Este método pode ajudar a entender mais efetivamente a inserção e a relação dos clientes no seu meio social. Também possibilita o acesso aos sentimentos e emoções que emergem deste sujeito nas suas relações sociais. Os aspectos técnicos da proposta estão presentes em inúmeras pesquisas que abordam a questão da identidade social. 2,9,10,11, 12

\section{APLICAÇÃO DO MÉTODO}

Tendo como base o referencial teórico-metodológico da Ego-ecológica, desenvolveu-se a tese de doutoramento intitulada "Identidade Social de Usuários, Familiares e Profissionais de um Centro de Atenção Psicossocial no Rio de Janeiro". ${ }^{12} 0$ estudo teve por objetivo conhecer a identidade dos usuários, familiares e profissionais que atuavam no cenário dos Centros de Atenção Psicossocial (CAPS) e conhecer os significados relacionados ao serviço. A pesquisa foi realizada com 11 usuários, 11 familiares e 12 profissionais. Esta abordagem teórico-metodológica permitiu conhecer a identidade do indivíduo, suas peculiaridades e sua realidade através da representação que os entrevistados possuíam acerca de si mesmo e de seus grupos de pertencimento. Permitiu compreender cada sujeito/identidade inserido em seu contexto, assim como as relações estabelecidas entre eles e seus pares e entre eles e os sujeitos dos outros grupos com os quais se relacionava no cotidiano do atendimento no CAPS.

Através da aplicação do referido referencial teóricometodológico foi possível observar fatores facilitadores e limitadores. Entre os fatores facilitadores, destaca-se a possibilidade de o próprio entrevistado atribuir valor, assim como se incluir ou não nos eixos propostos (egomórfico ou alomórfico, percepção positiva ou negativa do aspecto relatado pelo entrevistado), reduzindo o número de inferências do pesquisador nos achados da pesquisa; é de fácil aplicação, pois há indicação da organização do instrumento no referencial teórico primário; permite conhecer a percepção que o entrevistado tem de si mesmo, assim como as relações que estabelece com o seu grupo de pertencimento e com os indivíduos de outros grupos com os quais convive.

A experiência de aplicação do referencial teóricometodológico da Ego-ecologia indicou poucos fatores limitadores; porém, vale a pena ressaltar que o entrevistado precisa estar apto a valorar os aspectos citados por ele mesmo. No desenrolar das entrevistas, ao aplicar o instrumento, observou-se que pessoas com baixa escolaridade tiveram muita dificuldade em responder e classificar suas respostas.

No que diz respeito ao tempo de aplicação da entrevista, percebeu-se que é extremamente variável. Houve entrevistas de cerca de trinta minutos; porém, algumas chegaram a noventa minutos. Observou-se que o instrumento permite que o participante da pesquisa discorra sobre sua história de vida, suas angústias e expectativas e suas dificuldades em lidar com seus pares e com os grupos com quem convive, sendo possível observar as relações de conflito existentes no contexto estudado. 
A utilização do referido referencial teóricometodológico mostrou-se extremamente eficaz no seu propósito, possibilitando discussão crítica e profunda a respeito da relação entre usuários, seus familiares e os profissionais, de suas perspectivas para o futuro e sua visão do serviço em que foi realizado o estudo.

\section{REFLEXÕES FINAIS}

Como foi discutido anteriormente, a pesquisa em enfermagem vem modificando o seu objeto de interesse, seus referenciais teóricos e metodológicos, adotando contribuiç̃es oriundas das áreas sociais e filosóficas. 0 foco de atenção dos enfermeiros tem ampliado sua perspectiva a respeito do sujeito de seu cuidado, abordando inclusive o ambiente no qual ele se insere e as relações que estabelece. Portanto, para se compreender e incrementar o cuidado de enfermagem utilizam-se abordagens que vão além das ciências biológicas. A enfermagem emprega conceitos e conhecimentos também da antropologia, da sociologia, da filosofia e da história, pois

\begin{abstract}
"se o cuidado é a natureza viva do pensar, é preciso saber como ele se dá no seu campo de ação, o que produz nos clientes quando são tocados por ele e o que significa pensar nele... associando o que é preciso (ciência vigente) com o impreciso (as ciências que envolvem o social e a emoção), o quantitativo com o qualitativo, $o$ mensurável com o imensurável, o racional com o

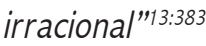

Neste sentido, através do referencial da Ego-ecologia é possível compreender de forma mais clara o indivíduo, seu ambiente e suas relações. 0 referido referencial teóricometodológico favorece a busca de informações para compreender o sujeito que está sendo cuidado, assim como o ambiente em que está acontecendo o cuidado, seu ambiente social e as relações que se dão no cotidiano. A análise ego-ecológica permite compreender mais amplamente o cliente dentro de sua complexidade e paradoxos, bem como as relações que estabelece no contexto da instituição, na família, no trabalho e demais situaç̃̃es sociais relevantes para a vida do cliente, quer estejam localizadas no espaço micro ou macrossocial.

0 referencial teórico-metodológico da Ego-ecologia indica estratégia alternativa para o desenvolvimento de pesquisas que discutam a identidade do sujeito e suas aproximações e afastamentos dos grupos dos quais faz parte. Desta forma, este referencial pode ser uma ferramenta interessante nas pesquisas em enfermagem, pois permite conhecer de forma profunda a identidade pessoal, bem como da identidade social dos sujeitos que se propõe a estudar.

A análise ego-ecológica pode ser útil aos enfermeiros, pois atende às necessidades dos pesquisadores que buscam compreender melhor 0 objeto de sua ação (pessoa que precisa de ajuda) e o objeto de sua intervenção (o cuidado). Além disso, propõe um método sistematizado, dinâmico e flexível para se compreender o indivíduo, seu contexto social e sua relação com seus grupos de pertencimento.

\section{REFERÊNCIAS}

1.Santos I. Cientificidade na enfermagem: uma ideologia compartilhada. Rev Enferm UERJ. 1998 jun; 6(1): 215-21.

2.Zavalloni M, Louis-Guérin, C. Identité sociale et conscience. Introduction à l'égo-écologie. Montreal: Lês Presses de L'Université de Montreal; 1984.

3.Santos TCF, Gomes MLB. Nexos entre pós-graduação e pesquisa em Enfermagem no Brasil. Rev Bras Enferm. [on-line] 2007; [citado 2011 mar 22]; 60(1): [aprox 5 telas]. Disponível em: http:// www.scielo.br

4.Associação Brasileira de Enfermagem- ABEn. Levantamento de recursos e necessidades de enfermagem no Brasil. 0 compromisso histórico da ABEn no desenvolvimento da pesquisa e da produção de conhecimento. [citado 2011 mar 22]; [aprox. 2 telas] Disponível em: http://www.abennacional.org.br/centrodememoria/ levantamentoderecursos.pdf

5.Moriya TM, Furegato AR, Almeida MCP, Ruffino MC, Oliveira MHP. Pós-Graduação "stricto sensu" Em enfermagem: um estudo do seu desenvolvimento. Ribeirão Preto: Fundação Instituto de Enfermagem; 1998.

6Moura MAV, Spindola T, Ferrer GH, Siqueira PRA, Chamilco RA. Tendências da produção de enfermagem na área da saúde da mulher. Esc Anna Nery. 2001 dez; 5(3): 335-46.

7.Alvim NAT, Cabral IE, Soares CMJ, Vargas ARGAS MÑ. 0 espaço criativo e sensível na produção de dados para a pesquisa em enfermagem. Esc Anna Nery. 2001 ago; 5(2): 191-99.

8.Ferreira MA. A comunicação no cuidado: uma questão fundamental na enfermagem. Rev Bras Enferm. 2006 maio/jun; 59 (3): 327-30.

9.Zavalloni, M. Subjective culture, self concept and the social environment. Intern J Psychol. 1973; 8(3): 183-92. 
10.Doise W, Zavalloni M. The generality of social perception characteristics. Acta Psychol. 1970; 34: 521-24.

11. Zavalloni M. Cognitive process and social identity through focused introspection. European J Social Psychol. 1971 Apr/Jun; 1(2): 23560.

12.Mello R. Identidade social de usuários, familiares e profissionais de um centro de atenção psicossocial no Rio de Janeiro [tese]. Ribeirão Preto: Escola de Enfermagem de Ribeirão Preto, Universidadede São Paulo; 2005.

13.Figueiredo NMA, Machado WCA. Cuidado: a natureza viva do pensar e do fazer. Esc Anna Nery. 2001 dez; 5(3): 377-86. 